\title{
Use of spectral decomposition to detect hydrocarbon-related dispersion anomalies.
}

\author{
Mark Chapman, Enru Liu \& Jinghua Zhang. \\ Edinburgh Anisotropy Project, British Geological Survey, West Mains Road, Edinburgh, EH9 \\ 3LA, UNITED KINGDOM. \\ Email: mhch@bgs.ac.uk
}

For many years, geophysicists have been intrigued by the possibility of using attenuation measurements to discriminate different fluid saturations. The basis of the attempts has been the observation from laboratory data that the saturating fluid plays a controlling role in attenuation measurements. Nevertheless, attempts to apply the techniques in practice have been disappointing, and numerical studies have demonstrated that the effect of attenuation should only be detectable in specialized circumstances.

Recent work on the application of spectral decomposition to seismic data has refocused attention on the long standing suspicion that reflections from hydrocarbon-bearing zones typically display systematic frequency anomalies. Many potential explanations have been advanced for such effects, but a definitive theory is not available and routine modeling of the phenomenon is generally not possible.

We review the rock-physics mechanisms which can explain the observed link between fluid saturation and seismic attenuation. These mechanisms generally predict that the attenuation is accompanied by significant velocity dispersion. The influence of this velocity dispersion is ignored in most synthetic modeling exercises, which usually treat attenuation by introducing a frequency-independent imaginary part to the elastic tensor.

Synthetic seismograms which account for the influence of both the fluid-related attenuation and velocity dispersion can be calculated using the reflectivity method with frequency-dependent elastic tensors. These synthetic seismograms demonstrate the existence of a strongly frequencydependent reflection coefficient for interfaces between layers with water saturation, which show weak attenuation, and layers with hydrocarbon saturation with strong attenuation and velocity dispersion. The effect of this is that reflections from such an interface are "shifted" to lower or higher frequency, with the direction and magnitude of the shift being controlled by the AVO behaviour at the interface. These shifts can be demonstrated particularly clearly by applying spectral decomposition techniques to the synthetic seismograms. Experience of this type of analysis has motivated the development of spectral decomposition based techniques which can illuminate such “dispersion anomalies”.

We have applied such techniques to seismic reflection data from a number of areas. Our approach begins with standard AVO analysis, and computation of synthetic seismograms using the Gassmann theory for fluid replacement modeling. This allows us to assess the effect of fluidsensitive tuning on the spectral response, but in general we find that this effect cannot by itself explain the frequency-dependent reflectivity which we observe in our data. The introduction of dispersion into the modeling allows us to match the spectral behaviour of the synthetics to that of the data. In this way, we show that the identification of zones of anomalously high dispersion can give insight into fluid saturation. 